\title{
Inelastic focusing effects in atom-surface scattering
}

\author{
A. Glebov, J. R. Manson, ${ }^{*}$ S. Miret-Artés, ${ }^{\dagger}$ J. G. Skofronick, ${ }^{\ddagger}$ and J. P. Toennies \\ Max-Planck-Institut für Strömungsforschung, Bunsenstraße 10, Göttingen, Germany
}

(Received 29 September 1997)

\begin{abstract}
An inelastic focusing mechanism has been identified in the angular distributions of helium atoms scattered from single-crystal $\mathrm{NaCl}(001)$ surfaces as being due to energy exchange with a Rayleigh phonon mode. This effect, called inelastic focusing, occurs under special conditions in which the small spread of energies in the incident beam is sharply focused into a very narrow range of final angles. For a fixed initial scattering angle this is mathematically described as a singularity associated with the density of states in the incident momentum as a function of final scattering angle. This effect is an intrinsic feature of any scattering event of particles from surfaces and could be used to greatly enhance the intensity of weak single-phonon features in inelastic timeof-flight spectra. [S0163-1829(98)50416-X]
\end{abstract}

The scattering of atoms and molecules from single-crystal surfaces has provided a wealth of information both on the physical properties of surfaces ${ }^{1}$ and also on the interactions of the particles with the surface. Initially, most experiments concentrated on diffraction studies of the surface structure, but during the last two decades, the development of highintensity nearly monoenergetic helium-atom beams has provided sufficiently high intensities so that dynamical features of the surface such as phonon dispersion curves could also be explored. $^{2}$ In the ensuing period many of the unexpected features observed in these measurements ${ }^{2-6}$ were explained in terms of selective adsorption resonances, both elastic and those involving phonons, as well as kinematic focusing. ${ }^{7}$ More recently, focused inelastic resonance (FIR) (Refs. 8 and 9) and diffuse elastic ${ }^{10}$ resonance (DER) effects have also been identified as causing distinct features in heliumatom scattering experiments. Since the interaction potential between $\mathrm{He}$ atoms and the $\mathrm{NaCl}(001)$ surface is reasonably well known, ${ }^{9-11}$ many of these new resonance mechanisms and focusing phenomena have first been studied on this surface.

In parallel with experiments, the theory of atom-surface scattering has also undergone significant development. However, the energy resolution of the incident He-atom beam has usually been considered to have no substantial effect on the distribution of the scattering intensity. Thus, for example, in the calculations of the differential reflection coefficients for elastic and inelastic scattering the incident beam of the He atoms is usually considered to be sharp in both energy and angles, i.e., the finite energetic width of the incident beam is neglected and it is usually represented by a well-defined plane wave. In practice, this would appear to be justified since in most $\mathrm{He}$-atom scattering experiments the energy resolution is usually as small as $\Delta E_{i} / E_{i} \approx 2 \%$.

If the finite angular width of the incident beam is included in the calculations several singularities appear that strongly modify the final scattering intensity. One of these new singularities, the inelastic focusing (IF) singularity discussed here, was observed previously in the rotationally inelastic diffraction of molecular beams of $\mathrm{H}_{2}$ and its isotopes from single crystals of $\mathrm{MgO}(001)$ (Ref. 12) as well as in the inelastic scattering of $\mathrm{D}_{2}$ from $\mathrm{NaF}(001) .{ }^{13}$ In this communi- cation, experimental evidence for the atomic IF effect in the scattering of $\mathrm{He}$ from $\mathrm{NaCl}(001)$ is presented. Moreover, in atomic scattering from other surfaces such as, for example, $\mathrm{He} / \mathrm{LiF}(001){ }^{6}$ certain features were previously observed that can now be attributed to IF. In this work, the IF singularity is reexamined and found to have more far-reaching physical implications than previously envisaged. We note that the IF is a quite distinct effect from the previously investigated FIR (Ref. 9) and DER (Ref. 10) resonances. Both FIR and DER involve a resonant transition through a bound state of the interaction potential, whereas the IF is a kinematic enhancement of any selected inelastic feature and does not require the presence of a bound state.

To experimentally demonstrate the IF singularity, highresolution helium-atom scattering experiments were carried on the well-studied $\mathrm{NaCl}(001)$ surface. In the scattering apparatus, which has been described in detail previously, ${ }^{14} \mathrm{a}$ nearly monoenergetic helium-atom beam $\left(\Delta E_{i} / E_{i} \leqslant 2 \%\right)$ is scattered through a fixed angle of $90^{\circ}$ between source and detector in a plane perpendicular to the crystal surface. The parallel wave-vector transfer $\Delta K$ is changed by rotating the crystal and is given by

$$
\Delta K=K_{f}-K_{i}=k_{f} \sin \theta_{f}-k_{i} \sin \theta_{i}
$$

where $k_{i}$ and $k_{f}$ are the incident and final wave vectors and their respective angles measured with respect to the surface normal are $\theta_{i}$ and $\theta_{f}$. The energy transfer is given by

$$
\Delta E=E_{f}-E_{i}=k_{f}^{2}-k_{i}^{2}
$$

in units of $\hbar^{2} / 2 m=1$ where $m$ is the mass of the helium atom. The energy transfer is measured by chopping the incident beam and measuring the time-of-flight distribution of the scattered atom. For a given set of initial conditions $k_{i}$ and $\theta_{i}$ the accessible values of $\Delta E$ as a function of $\Delta K$ can be calculated from Eqs. (1) and (2), which defines the scan curve: 


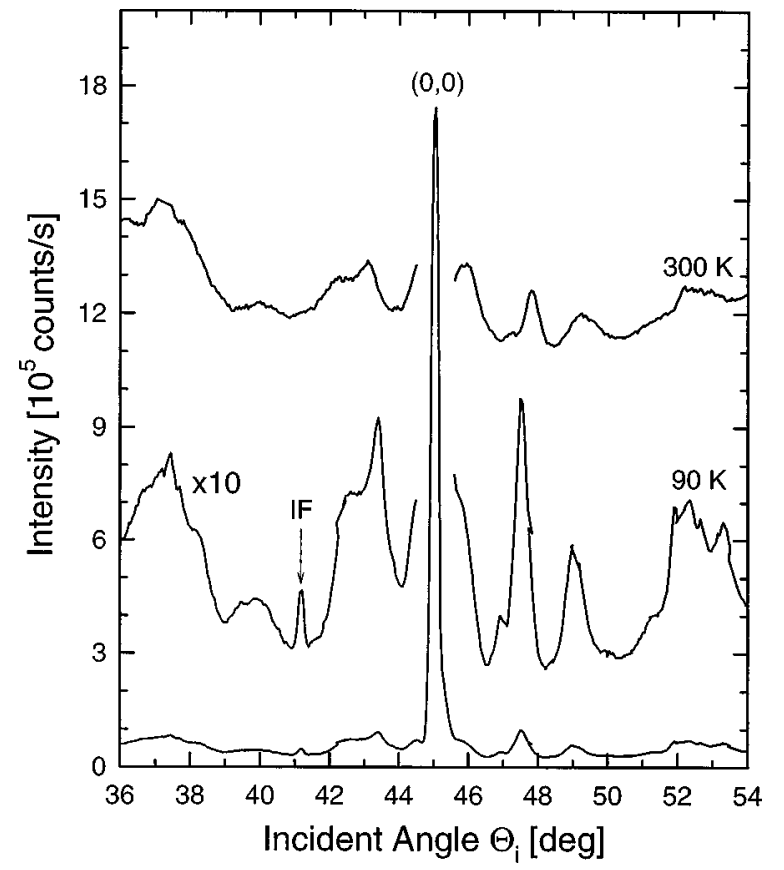

FIG. 1. The angular distribution of He atoms with wave vector $k_{i}=5.85 \AA^{-1}$ incident on the $\mathrm{NaCl}(001)[110]$ surface $\left(T_{S}=90 \mathrm{~K}\right)$ showing both a distribution with the large $(0,0)$ specular peak, and an expanded version showing several smaller peaks in the background. Most of the peaks have been previously assigned to phonon assisted selective adsorption and desorption resonances. The peak labeled IF is narrow in angle; it cannot be fitted to any known resonance condition and, therefore, is a candidate for the inelastic focusing effect described in this work. For comparison we also show the angular distribution measured (Ref. 8) under identical conditions but at $T_{S}=300 \mathrm{~K}$.

$$
\sin \theta_{f}=\frac{k_{i} \sin \theta_{i}+\Delta K}{\sqrt{k_{i}^{2}+\Delta E}} .
$$

The experiments were carried out for incident energies ranging between $E_{i}=10$ and $20 \mathrm{meV}$. The $\mathrm{NaCl}$ crystal (10 $\times 10 \times 10 \mathrm{~mm}^{3}$ ) was cleaved in vacuum at $p<10^{-10}$ mbar. The crystal temperature was $90 \mathrm{~K}$, which is sufficiently low for the multiphonon inelastic background to be small but high enough that there is no appreciable adsorption of residual gases on the surface, as evidenced by the fact that during an entire set of measurements, which takes approximately $5 \mathrm{~h}$, there are no detectable changes in the intensities.

Figure 1 shows an angular distribution for $\mathrm{NaCl}(001)[110]$ where one of the peaks (labeled IF) has some special features: (i) its angular position cannot be fitted by the usual known resonance and kinematic processes and (ii) the peak is narrower than the other observed features. These properties made this peak appear to be a likely example of the IF effect. To further strengthen this assignment several angular distributions were measured for a range of incident wave vectors $k_{i}$ between 5.56 and $6.13 \AA^{-1}$ as shown in Fig. 2 over the range of incident angles between $35^{\circ}$ and $50^{\circ}$. The peak occurring at essentially the same incident angle (and thus also the same final scattering angle) $\theta_{i}=41.1^{\circ}$ is observed in the whole range of incident wave vectors plotted in that figure. At 5.56, 5.64, and $5.71 \AA^{-1}$ the peak is barely visible. This peak initially grows as $k_{i}$ in-

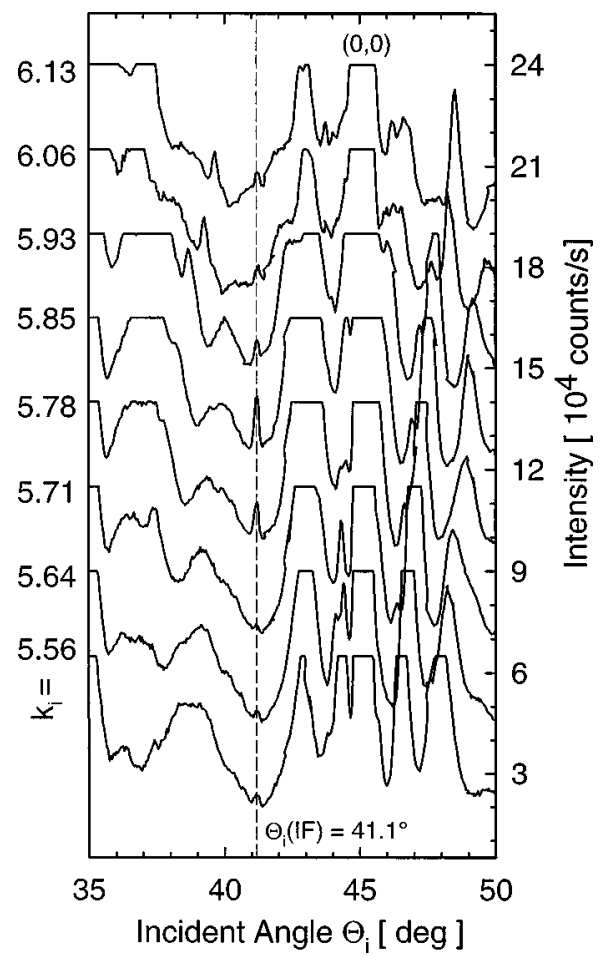

FIG. 2. Eight angular distributions for $\mathrm{He}$ atoms with wave vectors from $k_{i}=5.56$ to $k_{i}=6.13 \AA^{-1}$ incident on the $\mathrm{NaCl}(001)$ [110] surface. The peaks lying under the vertical dashed line and at the same incident angle $\theta_{i}=41.1^{\circ}$ initially grow in intensity as the incident wave vector increases and then decrease beyond the maximum at $k_{i}=5.85 \AA^{-1}$. This behavior is expected for the IF effect described in this work.

creases from the lowest value, becoming a maximum at $5.85 \AA^{-1}$ and then decreases again as $k_{i}$ is further increased. This indicates that the focusing mechanism takes place at $\theta_{i}=41.1^{\circ}$ and $k_{i}=5.85 \AA^{-1}$.

The origin of this special peak is shown in Fig. 3 in which the solid line $a$ shows, for fixed $\theta_{i}=41.1^{\circ}, \theta_{f}$ plotted against $k_{i} \quad$ using the scan curve (3) with $(\Delta E, \Delta K)$ $=\left(5.62 \mathrm{meV}, 1.21 \AA^{-1}\right)$. The extremum condition occurring at $\theta_{f}=90^{\circ}-\theta_{i}=48.9^{\circ}$ and $k_{i}=5.85 \AA^{-1}$ is evident. Because these particular values of $\Delta E$ and $\Delta K$ happen to fall on a point of the Rayleigh mode dispersion curve, the extremum amplifies the intensity at this point. As seen in Fig. 3, the extremum condition is obtained for a large range of $k_{i}$ vectors between approximately 5 and $7 \AA^{-1}$. Shown also in Fig. 3 as dash-dot curves are two other examples of this effect that correspond to points on the same Rayleigh phonon dispersion curve: $b$ for $(\Delta E, \Delta K)=\left(4.18 \mathrm{meV}, 1.3 \AA^{-1}\right)$ at $\theta_{i}=38.9^{\circ}$, and $c$ for $(\Delta E, \Delta K)=\left(8.55 \mathrm{meV}, 1 \AA^{-1}\right)$ at $\theta_{i}=43.3^{\circ}$. These two additional curves illustrate the fact that, with proper choice of $k_{i}$ and $\theta_{i}$, the singularity can be placed at any point in the $(\Delta E, \Delta K)$ plane. However, when the singular point coincides with an observable feature in the phonon spectrum, this results in an enhancement of intensity, as shown below.

The total in-plane scattering intensity observed experimentally in angular distributions, when a spread of incident energies and angles is present in the incident beam, can be expressed in general as 


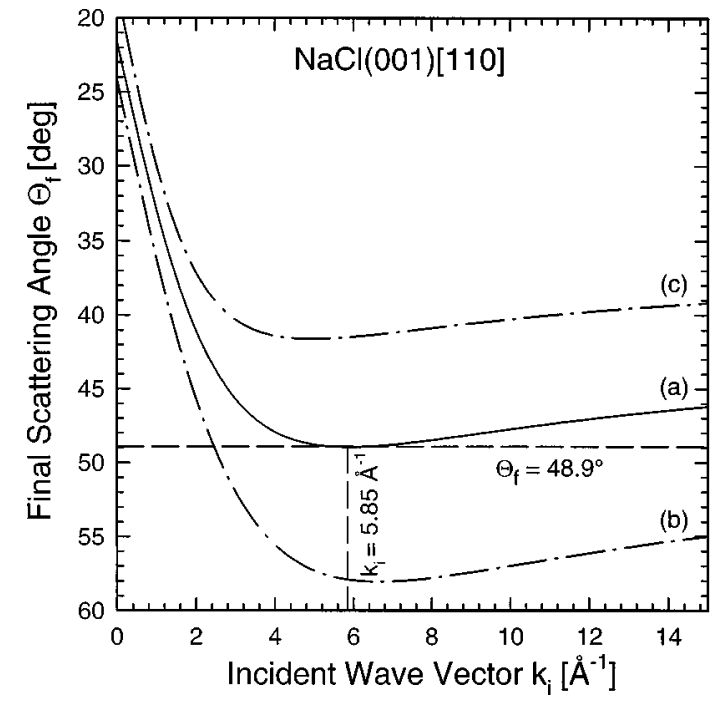

FIG. 3. For the $\mathrm{NaCl}(001)$ [110] surface, a plot of the scan curve of Eq. (3) expressed in terms of the final scattering angle $\theta_{f}$ vs the incident wave vector $k_{i}$. The solid curve labeled $a$ shows for the measurements in Fig. 2 that the IF condition is met for $\theta_{f}=48.9^{\circ}$ when the incident angle $\theta_{i}$ is fixed at the value of $41.1^{\circ}$ and $k_{i}$ $=5.85 \AA^{-1}$ with $(\Delta E, \Delta K)=\left(5.62 \mathrm{meV}, 1.21 \AA^{-1}\right)$. The dashdot curves show two other possible examples of focusing involving phonons on the same Rayleigh dispersion curve at $b \theta_{i}=38.9^{\circ}$ and $(\Delta E, \Delta K)=\left(4.18 \mathrm{meV}, 1.3 \AA^{-1}\right), \quad$ and at $c \quad \theta_{i}=43.3^{\circ}$ and $(\Delta E, \Delta K)=\left(8.55 \mathrm{meV}, 1 \AA^{-1}\right)$.

$$
\begin{aligned}
\frac{\Delta I\left(\mathbf{k}_{i 0}\right)}{\Delta E_{f} \Delta \theta_{f, \mathrm{det}}} \propto \int_{\Delta k_{i}} d k_{i} \rho\left(\mathbf{k}_{i}, \mathbf{k}_{i 0}\right) \int_{\Delta \theta_{f, \mathrm{det}}} d \theta_{f}\left(\frac{\partial k_{i}}{\partial \theta_{f}}\right)_{\theta_{i}, \Delta E_{0}, \Delta K_{0}} \\
\quad \times \int_{\Delta E_{f}} d E_{f} \frac{d R\left(\mathbf{k}_{f}, \mathbf{k}_{i}\right)}{d E_{f} d k_{i}}
\end{aligned}
$$

where the integrations are performed over all the incident phase space (energies and angles) in the incident beam and over the angular acceptance of the detector, respectively; $\rho\left(\mathbf{k}_{i}, \mathbf{k}_{i 0}\right)$ is the incident beam distribution around the central value of $\mathbf{k}_{i 0}$ and $d R\left(\mathbf{k}_{f}, \mathbf{k}_{i}\right) / d E_{f} d \theta_{f}=\left[d R\left(\mathbf{k}_{f}, \mathbf{k}_{i}\right) /\right.$ $\left.d E_{f} d k_{i}\right]\left[d k_{i} / d \theta_{f}\right]$ is the total differential reflection coefficient. Finally, $\left(\partial k_{i} / \partial \theta_{f}\right)_{\theta_{i}, \Delta E_{0}, \Delta K_{0}}$ is the density of states of $k_{i}$ for a given $\theta_{f}$ and a fixed $\theta_{i}$ and for fixed values of ( $\Delta E, \Delta K)$ denoted by $\left(\Delta E_{0}, \Delta K_{0}\right)$. The IF singularity in this type of scattering occurs when this density of states becomes very large. However, the corresponding divergence is integrable. In the following, the kinematics of this singularity is illustrated for a particular case.

The condition for a singularity in the density of states, $\left(\partial \theta_{f} / \partial k_{i}\right)_{\theta_{i}, \Delta E_{0}, \Delta K_{0}}=0$, can be expressed using Eq. (3) as

$$
k_{i}=\frac{\Delta E_{0}}{\Delta K_{0}} \sin \theta_{i}
$$

which can also be written as $k_{f} \sin \theta_{i}=k_{i} \sin \theta_{f}$. An alternative expression is obtained by combining the condition (5) with the scan curve (3) which produces the IF locus condition, expressed parametrically as

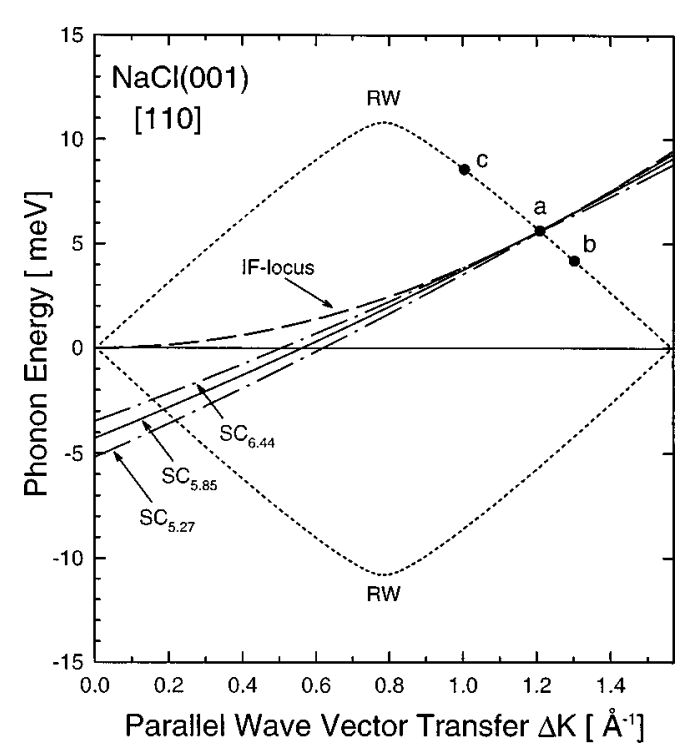

FIG. 4. For the $\mathrm{NaCl}(001)$ [110] surface, the short-dashed curve is a plot of the Rayleigh mode dispersion curve. The solid line is the scan curve for $\theta_{i}=41.1^{\circ}$ and $k_{i}=5.85 \AA^{-1}$, and the dashed line is the IF locus condition of Eq. (5). This illustrates the common tangency of the IF locus and scan curves that coincide at the point $a$ with $(\Delta E, \Delta K)=\left(5.62 \mathrm{meV}, 1.21 \AA^{-1}\right)$ on the Rayleigh mode. This corresponds to the IF enhancement shown in Figs. 1 and 2. The bunching of the scan curves at the singular point $a$ is shown by the dash-dot curves, which are scan curves calculated for $k_{i}=5.27$ and $6.44 \AA^{-1}$. The points $b$ and $c$ on the Rayleigh dispersion curve refer to the corresponding cases in Fig. 3 .

$$
\begin{aligned}
& \Delta E_{0}\left(k_{i}\right)=k_{i}^{2} \frac{\sin ^{2} \theta_{f}-\sin ^{2} \theta_{i}}{\sin ^{2} \theta_{i}} \rightarrow k_{i}^{2} \frac{\cos 2 \theta_{i}}{\sin ^{2} \theta_{i}}, \\
& \Delta K_{0}\left(k_{i}\right)=k_{i} \frac{\sin ^{2} \theta_{f}-\sin ^{2} \theta_{i}}{\sin \theta_{i}} \rightarrow k_{i} \frac{\cos 2 \theta_{i}}{\sin \theta_{i}},
\end{aligned}
$$

where the final expressions on the extreme right-hand side of Eq. (6) are the special cases for a fixed source-detector angle of $\theta_{f}+\theta_{i}=90^{\circ}$. This shows that the IF locus occurs at a single $\left(\Delta E_{0}, \Delta K_{0}\right)$ value for each $k_{i}$ and $\theta_{i}$, and the energy and momentum exchange at the IF point are quadratically related according to $\Delta E_{0}\left(k_{i}\right)=\Delta K_{0}^{2}\left(k_{i}\right) /\left(\sin ^{2} \theta_{f}-\sin ^{2} \theta_{i}\right)$. If the point $\left(\Delta E_{0}, \Delta K_{0}\right)$ given by Eqs. (5) or (6) is chosen to coincide with a point on the phonon dispersion curve, then the intensity of that mode will be greatly enhanced due to the large density of states.

Moreover, this locus is a condition for the bunching of scan curves around the same values of energy and momentum exchange (the bunching point). ${ }^{9}$ For example, the condition $\left(\partial \Delta E / \partial k_{i}\right)_{\Delta K, \theta_{f}, \theta_{i}}=0$ leads to the same condition as Eq. (5), and is one way of physically fulfilling the condition of common intersection of all scan curves for a range of $k_{i}$ at the same point in the $(\Delta E, \Delta K)$ plane. This is illustrated in Fig. 4 where the IF locus corresponding to $\theta_{i}=41.1^{\circ}$ is shown together with the Rayleigh wave (RW) dispersion curve in the $(\Delta E, \Delta K)$ plane. The short-dashed lines correspond to the RW dispersion curves, the solid line corresponds to the scan curve for $\theta_{i}=41.1^{\circ}$ and $k_{i}=5.85 \AA^{-1}$, and the dashed line is the IF locus, Eq. (5). The point where the IF locus crosses the RW dispersion curve $\left(\Delta E_{0}\right.$ 
$=5.62 \mathrm{meV}, \Delta K_{0}=1.21 \AA^{-1}$ ) corresponds to the IF peak for the incident conditions of the scan curve. As can be seen, the IF locus is also tangent to the scan curve and the point of tangency is on the RW dispersion curve at $\left(\Delta E_{0}, \Delta K_{0}\right)$. The region of concatenation is large, and consequently the focusing condition is fulfilled for a wide range of incident wave vectors. This is seen in the two additional scan curves shown as dash-dot curves in Fig. 4 with $k_{i}=5.27$ and $6.44 \AA^{-1}$, a range of $k_{i}$ substantially larger than that of the series of angular distributions shown in Fig. 2. In spite of having a 20\% difference of incident momentum all of these scan curves very nearly pass through the same point, and this implies that all $k_{i}$ in the much narrower incident beam distribution will have scan curves that pass through this same point.

Thus it is possible to take advantage of this bunching condition by changing the incident conditions to access all points in the $(\Delta E, \Delta K)$ plane and thereby scan the dispersion curves of the different acoustical and optical modes. Furthermore, under special circumstances it is possible to choose the bunching point to be tangent to the Rayleigh dispersion curve and therefore to meet the condition for the kinematic focusing effect. ${ }^{7,9}$ This fact was observed and reported in Ref. 9 in a different context.

There are several important properties that arise when conditions are chosen such that the focus point coincides with a phonon mode. (1) Because the entire narrow energy distribution of the incident beam is focused at a single point, the energy resolution of the incident beam does not affect the angular width of an observable IF feature. Thus the angular width of an IF peak can be used to estimate the energy width (or natural lifetime) of phonon modes in a manner which is unaffected by the energy broadening of the beam. (2) The improved energy resolution under IF conditions should be of use for the measurement of phonons with other atomic or molecular probes which, as compared to He beams, often have a much broader spread of energies in the incident beam. ${ }^{6}$ (3) In principle, the sharp and distinctive signature of the focus effect, as exhibited in Figs. 1 and 2, will allow the measurement of phonon dispersion curves directly from the angular distributions without need for energy resolution of the final scattered beam. (4) This mechanism can be used to enhance very weak phonon features as for example occurs with adsorbate vibrational modes at very low coverages. (5) It could be used to enhance and distinguish fine structures in the phonon spectra such as nearly coinciding peaks in the intensity or avoided crossings of modes in the dispersion curves. (6) Finally, since this is a pure kinematic effect, independent of the type of projectile or the nature of the surface, it is applicable to other surface scattering experiments such as low-energy electron diffraction (LEED) or molecular scattering.

This research was supported in part by DOE Grant No. DE-FG02-97ER45635, NSF Grant No. DMR 9419427, and NATO Grant No. GRG.961145. J.R.M. and J.G.S. thank Professor J. P. Toennies and the Max-Planck-Institut für Strömungsforschung for their hospitality during a part of this work. S.M.-A. gratefully acknowledges support from the Alexander von Humboldt Foundation.
*Permanent address: Department of Physics and Astronomy, Clemson University, Clemson, SC 29634.

†Permanent address: Instituto de Matemáticas y Física Fundamental (CSIC), Serrano 123, 28006 Madrid, Spain.

${ }^{*}$ Permanent address: Department of Physics, Florida State University, Tallahassee, FL 32306.

${ }^{1}$ Helium Atom Scattering from Surfaces, edited by E. Hulpke (Springer, Berlin, 1992).

${ }^{2}$ G. Brusdeylins, R. B. Doak, and J. P. Toennies, Phys. Rev. B 27, 3662 (1983).

${ }^{3}$ H. Hoinkes, Rev. Mod. Phys. 52, 933 (1980).

${ }^{4}$ D. Evans, V. Celli, G. Benedek, J. P. Toennies, and R. B. Doak, Phys. Rev. Lett. 50, 1854 (1993).

${ }^{5}$ G. Brusdeylins, R. B. Doak, and J. P. Toennies, J. Chem. Phys. 75, 1784 (1981).

${ }^{6}$ G. Lilienkamp and J. P. Toennies, Phys. Rev. B 26, 4752 (1982);

J. Chem. Phys. 78, 5210 (1983).

${ }^{7}$ G. Benedek, Phys. Rev. Lett. 35, 234 (1975); G. Benedek, G.
Brusdeylins, J. P. Toennies, and R. B. Doak, Phys. Rev. B 27, 2488 (1983).

${ }^{8}$ G. Benedek and S. Miret-Artés, Surf. Sci. Lett. 339, 935 (1995).

${ }^{9}$ G. Benedek, R. Gerlach, A. Glebov, G. Lange, S. Miret-Artés, J.

G. Skofronick, and J. P. Toennies, Phys. Rev. B 53, 11211 (1996).

${ }^{10}$ A. Glebov, J. R. Manson, J. G. Skofronick, and J. P. Toennies, Phys. Rev. Lett. 78, 1508 (1997).

${ }^{11}$ G. Benedek, A. Glebov, W. Silvestri, J. G. Skofronick, and J. P. Toennies, Surf. Sci. Lett. 381, L540 (1997).

${ }^{12}$ R. G. Rowe, L. Rathbun, and G. Ehrlich, Phys. Rev. Lett. 35, 1104 (1975); R. G. Rowe, and G. Ehrlich, J. Chem. Phys. 63, 4648 (1975).

${ }^{13}$ G. Brusdeylins and J. P. Toennies, Surf. Sci. 126, 647 (1983).

${ }^{14}$ J. P. Toennies and R. Vollmer, Phys. Rev. B 44, 9833 (1991).

${ }^{15}$ J. P. Toennies, in Surface Phonons, edited by W. Kress and F. W. de Wette (Springer, Berlin, 1990); G. Benedek, Phys. Rev. Lett. 35, 234 (1975). 